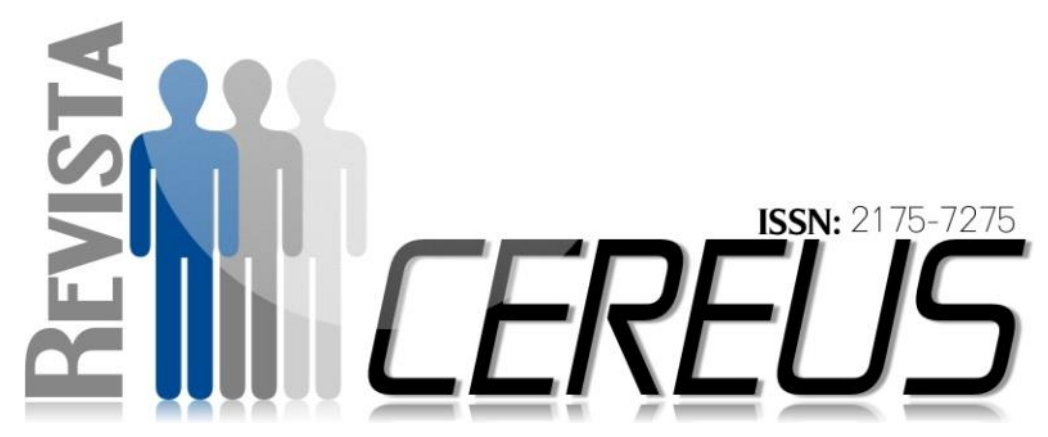

DOI:10.18605/2175-7275/cereus.v9n1p.18-36

\title{
EFICIÊNCIA NA ALOCAÇÃO DOS RECURSOS PÚBLICOS NA ÁREA DE SAÚDE: ANÁLISE DOS MUNICÍPIOS DE MINAS GERAIS
}

CARDOSO, Antônio Augusto Brion ${ }^{1}$

REIS, Anderson de Oliveira ${ }^{2}$

CASTRO, Elizangela Lourdes de ${ }^{3}$

REIS, Vanessa de Oliveira ${ }^{4}$

\section{RESUMO}

Conforme disposto no texto da carta Magna, aos cidadãos é garantido direito a saúde, sendo dever do estado promovê-la. Assim, o presente trabalho objetivou analisar se os recursos públicos destinados a saúde pública foram empregados pelos gestores de forma eficiente. Para elaboração da pesquisa, foram utilizados os dados referentes aos anos de 2005 e 2010

${ }^{1}$ Mestre em Administração (UFLA). Professor do Departamento de Ciências Contábeis da Universidade Federal de Juiz de Fora - Campus Avançado de Governador Valadares (UFJF/GV). E mail para correspondência: augusto.brion@ufff.edu.br.

${ }^{2}$ Mestre em Administração (UFV). Professor do Departamento de Ciências Contábeis da Universidade Federal de Juiz de Fora - Campus Avançado de Governador Valadares (UFJF/GV).

${ }^{3}$ Mestre em Ciências Contábeis (FUCAPE/ES). Professor do Departamento de Ciências Contábeis da Universidade Federal de Juiz de Fora - Campus 
dos 853 municípios mineiros. Tais dados foram coletados por meio do software IMRS (Índice Mineiro de Responsabilidade Social), considerando os seguintes indicadores: gasto per capita com atividade de saúde, proporção de internações por diabetes mellitus e suas complicações, proporção de nascidos vivos com baixo peso, proporção da população atendida pelo Programa de Saúde da Família (PSF),cobertura vacinal de febre amarela em menores de 1 ano, proporção da população coberta por planos privados de saúde, proporção de óbito por causas mal definidas sem assistência médica e proporção das internações para o parto de pacientes do SUS encaminhadas para outro Município. Para obtenção dos resultados da pesquisa, foram utilizado os métodos: AED (Análise Exploratória de Dados), DEA (Análise Envoltória de Dados) e Teste $t$ para amostras emparelhadas. Como resultado, observou-se uma melhoria significativa de eficiência entre 2005 e 2010, o que leva a concluir que os municípios mineiros tem se preocupado com a eficiência na aplicação dos recursos voltados para a saúde com o passar dos anos.

Palavras-Chave: Eficiência. Saúde. Administração Pública

EFFICIENCY IN ALLOCATION OF PUBLIC RESOURCES IN HEALTH AREA: ANALYSIS OF MUNICIPALITIES OF MINAS GERAIS

\section{ABSTRACT}

As stated in the Magna letter, citizens are guaranteed the right to health, and it is the duty of the state to promote it. Thus, the present study aimed to analyze whether public resources destined to public health were used by managers in an efficient way. For the elaboration of the research, the data referring to the years 2005 and 2010 of the 853 municipalities of Minas Gerais were used. These data were collected through the IMRS software (Social Responsibility Index in Minas), considering the 
following indicators: per capita expenditure with health activity, proportion of hospitalizations for diabetes mellitus and their complications, proportion of live births with low weight, proportion of Population covered by the Family Health Program (PSF), yellow fever vaccination coverage in children under 1 year of age, proportion of the population covered by private health plans, proportion of deaths due to ill-defined causes without medical assistance, and proportion of hospitalizations for childbirth for SUS (Brazilian health system) patients referred to another municipality. To obtain the results of the research, the following methods were used: AED (Exploratory Data Analysis), DEA (Data Envelopment Analysis) and $t$ Test for paired samples. As a result, a significant improvement in efficiency was observed between 2005 and 2010, which leads to the conclusion that the municipalities of Minas Gerais have been concerned with the efficiency in the application of resources directed to health over the years.

Keywords: Efficiency. Health. Public administration 


\section{INTRODUÇÃO}

Historicamente, o sistema de saúde no Brasil vem passando por grandes transformações desde a edição da Lei Elói Chaves em 1923 que criou as Caixas de Pensão e Aposentadoria. Desde lá, o Estado brasileiro vem buscando uma forma de criar garantias de assistência médica aos contribuintes. Para Barros (2011) com a criação do Centro Brasileiro de Estudos de Saúde (CCBE) em 1976, no período da Reforma Sanitária, seus integrantes iniciaram 0 processo da luta pela democratização da saúde e da sociedade como um todo, dessa forma impulsionaram os primeiros passos para normatização de um sistema que assegurasse 0 direito à saúde. Dessa forma, considera-se que a Reforma Sanitária foi o marco inicial anterior à garantia do direito à saúde, apresentado pela Constituição Federal de 1988:

Art. 196: A saúde é direito de todos e dever do Estado, garantido mediante políticas sociais e econômicas que visem à redução do risco de doença e de outros agravos e ao acesso universal e igualitário às ações e serviços para sua promoção, proteção e recuperação. (BRASIL 1988, Art. 196).

De acordo com a mesma Constituição Federal, a prestação de serviços de saúde tornou-se competência dos municípios, mas estes teriam ajuda financeira do Estado e da União, sendo a última mais representativa no repasse dos recursos.

Art. 198. As ações e serviços públicos de saúde integram uma rede regionalizada e hierarquizada e constituem um sistema único organizada de forma descentralizada, com direção única em cada esfera de governo. (BRASIL, 1988, Art. 198).

Posterior à promulgação da Constituição, foi aprovada a Lei Federal no 8080/1990 que regulamenta - Sistema Único de Saúde (SUS) como sendo um sistema descentralizado que tem como objetivo tornar a qualidade dos serviços prestados mais eficientes, isto por não concentrar nas mãos dos demais gestores estaduais estas ações e, somente, cabendo a gestão municipal o gerenciamento e a prestação destes serviços. Kohama (2012) entende a administração descentralizada ou indireta como aquela atividade administrativa, caracterizada como serviço público ou de interesse público, transferida ou deslocada do Estado com intuito de proporcionar satisfação de seus fins administrativos. 
A Lei 8080/1990 em seu artigo 31 afirma que o Sistema Único de Saúde (SUS) será financiado através dos seguintes recursos:

O orçamento da seguridade social destinará ao Sistema Único de Saúde (SUS) de acordo com a receita estimada, os recursos necessários à realização de sua finalidade, previstos em proposta elaborada pela sua direção nacional, com a participação dos órgãos da Previdência Social e da Assistência Social, tendo em vista metas e prioridades estabelecidas na Lei de Diretrizes Orçamentárias. (BRASIL, 1990, art. 31).

A receita destinada ao Sistema Único de Saúde será transferida pelas autoridades responsáveis ao Fundo Nacional de Saúde (FNS), de acordo com a proporção das despesas prevista de cada área, correspondente as dotações consignadas no Orçamento da Seguridade Social (BRASIL, 1990).

De acordo com o que está disposto no artigo 195 da Lei $8080 / 1990$, para o financiamento da seguridade social, serão empregadas as contribuições arrecadadas da sociedade de forma direta e indireta mediante recursos provenientes dos orçamentos da União, dos Estados, do Distrito Federal e dos Municípios. Além das contribuições que são empregadas ao financiamento da seguridade social, de acordo com a Ementa Constitucional 29/2000 os Estados devem garantir $12 \%$ de suas receitas de impostos e de transferências e os Municípios 15\% para financiamento da saúde.

Como transferências, a União garante aos Estados 25\% do produto de arrecadação dos impostos e aos Municípios, 50\% do produto da arrecadação do imposto da União sobre a propriedade territorial rural e $50 \%$ do produto da arrecadação do imposto do Estado sobre a propriedade de veículos automotores licenciados em seus territórios (BRASIL, 1990). Ademais, na hipótese do SUS não financiar a necessidade dos cidadãos, o setor privado pode atuar de forma complementar por meio de contratos e convênios de prestação de serviço ao Estado.

Para que os recursos sejam empregados atendendo a necessidade da população, o gestor deve seguir os pressupostos do artigo 37 da Constituição Federal, que afirma que a administração direta ou indireta de qualquer dos poderes da União deverá obedecer aos princípios de legalidade, impessoalidade, moralidade, publicidade e eficiência. Porém, é público e notório que os recursos 
destinados para a saúde são limitados, desta forma, sua alocação deve ocorrer de maneira ótima de modo que as necessidades de cada região sejam minimamente atendidas.

A mensuração da eficiência de saúde em países pobres como o Brasil torna-se fundamental, visto que não parece justo e tão pouco aceitável atender aos acréscimos da demanda social por serviços mediante somente do financiamento por mais impostos, sem ao menos pensar como esses recursos estão sendo alocados (MARINHO; FAÇANHA, 2001). Alcançar financiamento suficiente é imperativo, mas ter os recursos financeiros não assegura a cobertura universal. Assim, para que se possa garantir acesso a saúde de toda população é importante que os recursos sejam utilizados de maneira eficiente (OMS, 2010).

Com base no que foi exposto, o presente estudo se vê diante da seguinte questão: Os recursos destinados à área da saúde estão sendo alocados com eficiência nos municípios mineiros pelos gestores municipais? É sabido que de acordo com a Constituição federal de 1988, todos são iguais a luz da Lei, e ainda, onde é garantido tanto a brasileiros quanto aos estrangeiros residentes no País a inviolabilidade do direito à vida. Por tais aspectos, o presente estudo tem o objetivo de analisar se os recursos destinados à saúde estão sendo alocados com eficiência nos 853 municípios mineiros.

Dessa forma, objetivou-se especificamente: (i) analisar a alocação dos recursos públicos na área da saúde para cada município mineiro nos anos de 2005 e 2010; (ii) propor um ranqueamento dos municípios mineiros, por meio da análise de eficiência pelo método analise envoltória de dados; (iii) além de identificar o comportamento dos municípios com relação ao seu nível de eficiência nos anos 2005 e 2010. Estudos anteriores como o de Cabral e Ferreira (2014), buscou analisar os níveis de eficiência na utilização dos recursos de saúde com enfoque sobre - Pacto pela Saúde, analisando 30 municípios mineiros que aderiram ao pacto. Já Matta (2014) verificou o desempenho dos municípios na Estratégia Saúde da Família (ESF) em Minas Gerais através de indicadores de eficiência e resultados na Atenção Primaria à Saúde e investimentos nessa estratégia, por meio de gastos, número de equipes e número de 
Unidades Básicas de Saúde, que se relacionam com as metas da ESF.

Apesar dos avanços dos estudos anteriores, notou-se que eles apresentam enfoques específicos como o Pacto pela Saúde e a Atenção Primária a Saúde. Dessa forma, este estudo busca contribuir de forma

\section{MATERIAIS E MÉTODOS}

A metodologia adequada para o problema proposto é quantitativa e descritiva, onde os dados coletados têm como objetivo descrever as características de determinada população ou determinado fenômeno que são traduzidos em números para serem analisadas por métodos estatísticos. Marconi e Lakatos (2003) definem a pesquisa descritiva quantitativa como empírica, cuja finalidade é o delineamento ou análise das características de fatos, os fenômenos, a avaliação de programas ou isolamento de variáveis principais ou chave. Já Rampazzo (2005) define pesquisa descritiva como um fenômeno que observa, registra, analisa e correlaciona fatos e fenômenos (variáveis), ou seja, sem que haja a interferência do pesquisador. empírica para a literatura em Administração Pública brasileira, ao realizar uma análise global da eficiência na alocação de recursos à saúde, utilizando variáveis tanto da atenção primária (preventiva) como aquelas referentes a atenção secundária (paliativa).

O universo desta pesquisa são todos os municípios brasileiros, enquanto que a amostra a ser utilizada para elaboração do trabalho serão os municípios do estado de Minas Gerais. De acordo com Beuren (2008), as amostra são frações ou uma pequena parte dos elementos de um universo da pesquisa. O estado de Minas Gerais é dividido política e administrativamente em 853 municípios, que são subdivididos de acordo com o Instituto Brasileiro de Estatística e Geografia (IBGE) em 12 mesorregiões e 66 microrregiões.

A coleta de dados representa o meio pelo qual as informações sobre as variáveis são obtidas. Os dados coletados são secundários e foram obtidos por meio do Índice Mineiro de Responsabilidade Social - IMRS, que é um software que disponibiliza uma 
base de dados que além de atender às determinações da Lei № 15.011, de 15/01/2004, amplia informações para todos os Municípios Mineiros com confiabilidade, comparabilidade e periodicidade e são referentes às variáveis relativas à aplicação de recursos na área da saúde, nos anos de 2005 e 2010. Na primeira etapa do trabalho foi realizada a análise exploratória de dados (AED) com o objetivo de identificar as medidas de centro (mediana, média e moda), de dispersão (variância e desvio-padrão), outliers e a temporalidade dos dados que serão coletados para a análise da eficiência da gestão dos recursos da saúde.

A segunda etapa do trabalho referiu-se a análise da eficiência na aplicação dos recursos públicos na área da saúde, por meio do método denominado DEA (do inglês - Data EnvolopmentAnalysis), em português sua tradução é Análise Envoltória de Dados. Este método tem sido utilizado comumente para avaliar a eficiência da alocação dos recursos destinados aos Municípios e os serviços a eles prestados, além de medir a eficiência, proporcionando assim a identificação do desempenho da amostra analisada e a comparação entre elas (SILVA, 2009).

Para Ribeiro (2012), como ferramenta não paramétrica, a DEA avalia a eficiência técnica relativa às unidades produtivas, chamadas de Unidades Tomadoras de Decisões (DMU, do inglês DecisionMakingUnits) onde são comparadas entidades que realizam tarefas similares e se diferenciam pela quantidade de recursos utilizados (inputs) e de bens produzidos (outputs).

A mensuração dos escores de eficiência de acordo com Lins et al., (2007) é feita com base na seguinte fórmula:

Índice de Eficiência $=\frac{\Sigma_{j} U_{j} y_{k}}{\Sigma_{i} v_{i} x_{i k}}=\frac{u Y k}{v X k}$ Onde "u" e "v" são pesos ou multiplicadores e, por convenção $\frac{u Y k}{v X k}$ $\leq 1$, o que gera o índice de eficiência entre 0 e 1 .

De acordo com Lins et al., (2007):

"uma vez que a produção é um processo no qual os recursos $\left(X_{k}\right)$ são utilizados para gerar produtos $\left(Y_{k}\right)$, a fronteira de produtividade pode ser definida com a máxima quantidade de outputs obtida a partir dos inputs utilizados. A eficiência de cada DMU é a soma ponderada das entradas, a distribuição dos pesos ocorrendo sem qualquer interferência do decisor." (LINS et al., 2007). 
Como modelo de resultado da DEA, utilizou-se o CCR (Charnes, Cooper E Rhodes) ou CRS (Constant ReturnstoScale) que tange retornos de escalas constantes, ou seja, toda variação de inputs levará uma alteração proporcional aos outputs (MATTA, 2014).

Para execução do modelo DEA, anteriormente, foi realizada análise de cada DMU, restando o total de 256 municípios dos 853 propostos na pesquisa, tendo em vista que o modelo não aceita dados nulos ou faltosos, conforme os municípios excluídos apresentaram.

Como inputs (entrada de dados no sistema), foram utilizados a despesa na área de saúde realizada dos municípios, do período de 2005 e 2010, mais especificamente, os dados foram relativos ao gasto per capita com atividade de saúde. Foram utilizadas como outputs (produtos ou saídas de dados do sistema) as seguintes variáveis: estado da saúde, acesso a atenção básica e acesso ao atendimento médico-hospitalar.

Com relação ao Estado de Saúde, esta variável refere-se ao primeiro nível de atenção à saúde, segundo o modelo adotado pelo SUS, que garante a população acesso a especialidade básica, clínica médica (clínica geral), pediatria, obstetrícia e ginecologia. Neste estudo, foram utilizados os seguintes indicadores:

- Proporção de internações por diabetes mellitus e suas complicações; - Proporção de nascidos vivos com baixo peso;

Já o variável Acesso à Atenção Básica se refere ao primeiro nível de atendimento de atenção à saúde, onde a população tem acesso às especialidades básicas, e foi analisada a partir dos seguintes indicadores:

- Proporção da população atendida pelo Programa de Saúde da Família (PSF);

- Cobertura vacinal de febre amarela em menores de 1 ano;

O Acesso ao Atendimento Médico Hospitalar é uma variável que se refere à mensuração do atendimento fornecido à população, e foi analisada de acordo com os seguintes indicadores:

- Proporção da população coberta por planos privados de saúde; - $\quad$ Proporção de óbito por causas mal definidas sem assistência médica;

- $\quad$ Proporção das internações para - parto de pacientes do SUS encaminhada para outro Município.

A terceira etapa referiu-se à análise do comportamento dos 
municípios com relação ao seu nível de eficiência em 2005 e 2010. Para analisar as diferenças nos períodos analisados, utilizou-se $o$ teste $T$ para amostras emparelhadas, 0 qual permite inferir sobre a igualdade de médias de duas amostras emparelhadas (PESTANA; GAGEIRO, 2005).

\section{RESULTADOS E DISCUSSÃO}

\subsection{ANÁLISE EXPLORATÓRIA DE DADOS (AED)}

Com a análise exploratória dos dados foram coletadas as medidas de centro (média) e de dispersão (amplitude, desvio padrão) de cada
Para utilização do teste t para amostras emparelhadas foram necessárias a construção de duas hipóteses a serem testadas:

$\mathrm{H} 0=$ os valores das médias dos escores de eficiência são iguais para os dois períodos analisados;

$\mathrm{HA}=$ os valores das médias dos escores de eficiência são diferentes para os dois períodos analisados.

Tabela 1: Estatística descritiva 2005

\begin{tabular}{llllll}
\hline Variável & Média & Máximo & Mínimo & Amplitude & $\begin{array}{l}\text { Desvio } \\
\text { Padrão }\end{array}$ \\
\hline Gasto per capita & 148,73 & 485,78 & 3,88 & 481,90 & 67,52 \\
\hline Proplnt . D/M & 2,43 & 8,77 & 0,21 & 8,56 & 1,44 \\
\hline Prop.Nasc. B.P & 8,59 & 18,57 & 1,35 & 17,22 & 2,93 \\
\hline Prop.Pop.Atend. PSF & 62,48 & 100 & 1,38 & 98,62 & 27,19 \\
\hline Cob. Vac. F.A & 94,55 & 100 & 61 & 39 & 8,53 \\
\hline Prop. Pop. Cob. P. S & 6,52 & 40,78 & 0,01 & 40,77 & 8,48 \\
\hline Prop. Ob. S/M. & 14,11 & 55,56 & 0,09 & 55,47 & 14,4 \\
\hline Prop. SUS M & 45,51 & 100 & 0,10 & 99,90 & 44,39 \\
\hline
\end{tabular}

Com a análise descritiva dos dados, nota-se que o Gasto per capita em média em saúde foi de $R \$ 148,73$ e que $40,48 \%$ dos municípios estão acima da média. Delfinópolis atingiu o máximo na utilização do Gasto per capita com o valor de $R \$ 485,78$ e o indicador com o auxílio do software SPSS, sendo apresentados na tabela 1 os dados referentes a 2005 e na tabela 2 os dados referentes a 2010 . 
ao contrário de outros nos quais este gasto é muito abaixo do esperado.

Referindo-se à Proporção de internações por diabetes mellitus e suas complicações a média é de $2,43 \%$, sendo que $42,85 \%$ estão acima da média em relação aos demais municípios. Jequeri foi o município que mais obteve internações por diabetes mellitus e sua complicação, com $8,77 \%$, enquanto o menor valor foi apresentado pelo município Engenheiro de Caldas, com 0,21\%. Tendo em vista os valores de amplitude e desvio padrão, a proporção das internações por diabetes mellitus sofre grandes variações em relação à média.

A variável Proporção de nascidos vivos com baixo peso resultou na média de $8,59 \%$ em que $48,42 \%$ estão acima da média. Entre os municípios, Piedade de Ponte Nova é o que obteve maior proporção de nascidos vivos com baixo peso $(18,57 \%)$ e menor proporção em Entre Folhas 1,35\%. A grande variação entre os municípios pode ser evidenciada pelos valores de desvio padrão e amplitude, em que a proporção de nascidos vivos com baixo peso oscila consideravelmente em relação à média.
A Proporção da população atendida pelo Programa de Saúde da Família obteve média de $62,48 \%$. Dos 256 municípios analisados 28 alcançaram a maior proporção de atendimento, ou seja, $100 \%$, dentre eles podemos citar, Entre Folhas e Delfinópolis. O menor valor foi observado em Camanducaia, com $1,38 \%$. Os valores de amplitude e desvio padrão demonstram uma discrepância entre os municípios em relação da proporção da população atendida pelo Programa Saúde da Família.

A média de Cobertura vacinal de febre amarela em menores de 1 ano foi de $94,55 \%$, sendo que $67 \%$ dos municípios estão acima da média. Obtiveram cobertura máxima 145 municípios e mínima em três municípios com $61 \%$.

A Proporção da população coberta por planos privados de saúde tem como média $6,52 \%$, sendo que $31,34 \%$ dos municípios estão acima da média. Belo Horizonte é o município no qual $40,78 \%$ da população é coberta por planos privados de saúde, sendo esta a maior cobertura da amostra, enquanto Pintópolis e Verdelândia são os municípios com menor cobertura, a qual se limita a 
$0,01 \%$. Através dos valores de amplitude e desvio padrão demonstrase grande discrepância entre os municípios que a população é coberta por planos de saúde e os que não são.

Proporção de óbito por causas mal definidas sem assistência médica tem como média $14,11 \%$, sendo que $37,3 \%$ dos municípios estão acima da média. Alvorada de Minas apresentou maior proporção de óbito por causas mal definidas $(55,56 \%)$ e Sete Lagoas foi o que apresentou menor proporção $(0,09 \%)$. Através dos valores de amplitude e desvio padrão, demonstrase que existe grande discrepância entre os municípios que apresentam casos de óbito por causas mal definidas sem assistência médica em relação aos que não apresentam. Proporção das internações para 0 parto de pacientes do SUS encaminhados para outro município obteve como média 45,51\%, sendo que $41,27 \%$ estão acima da média. Dos 256 municípios analisados, 94 alcançaram o máximo de proporção das internações para parto de pacientes do SUS e o menor valor foi apresentado por Alfenas (0,10\%). São evidenciadas grandes variações com base na amplitude e desvio padrão entre os municípios. Para o ano de 2010, obteve-se:

Tabela 2: Estatística descritiva 2010

\begin{tabular}{llllll}
\hline \multicolumn{1}{c}{ Variável } & Média & Máximo & Mínimo & Amplitude & $\begin{array}{l}\text { Desvio } \\
\text { Padrão }\end{array}$ \\
\hline Gasto per capita & 318,99 & 827,40 & 105,11 & 722,29 & 103,02 \\
\hline PropInt . D/M & 4,34 & 21,41 &, 44 & 20,97 & 3,05 \\
\hline Prop.Nasc. B.P & 8,72 & 18,52 & 1,52 & 17,00 & 2,77 \\
\hline Prop.Pop.Atend. PSF & 84,24 & 100,00 & 13,31 & 86,69 & 437,87 \\
\hline Cob. Vac. F.A & 93,89 & 100,00 & 60,98 & 39,02 & 8,64981 \\
\hline Prop. Pop. Cob. P. S & 9,19 & 55,89 &, 10 & 55,79 & 11,07952 \\
\hline Prop. Ob. S/M. & 4,43 & 27,08 &, 12 & 26,96 & 4,43 \\
\hline Prop. SUS M & 49,61 & 100,00 &, 12 & 99,88 & 44,07 \\
\hline
\end{tabular}

Em relação ao Gasto per capita em saúde, a média dos municípios foi de $R \$ 318,99$, sendo que $41,40 \%$ dos municípios estão acima da média. Delfinópolis atingiu o máximo na utilização do Gasto per capita com o valor de $R \$ 827,40$ e o mínimo corresponde a Sabará com o valor de $R \$ 105,11$. Com base nos valores de amplitude e desvio padrão pode-se dizer que existem grandes variações entre os municípios, em que o gasto 
per capita de alguns é elevado ao contrário de outros que é muito abaixo do esperado.

Referindo-se a Proporção de internações por diabetes mellitus e suas complicações a média é de $4,34 \%$, sendo que $40,23 \%$ estão acima da média em relação aos demais municípios. Turmalina foi o Município que mais obteve internações por diabetes mellitus, com 21,41\%, enquanto 0 menor número de internações foi apresentado por Florestal, com 0,44\%. Tendo em vista os valores de amplitude e desvio padrão, a proporção das internações por diabetes mellitus sofreram grandes variações em relação à média.

A variável Proporção de nascidos vivos com baixo peso resultou na média de $8,72 \%$ em que $51,95 \%$ estão acima da média. Entre os municípios, Mesquita obteve $18,52 \%$ proporção de nascidos vivos com baixo peso e Fronteira dos Vales $1,52 \%$, o que se evidencia grande variação em relação à média de acordo com os valores de desvio padrão e amplitude.

A Proporção da população atendida pelo Programa de Saúde da Família obteve média de 84,24\%, sendo que $63,68 \%$ estão acima da média. Dos 256 municípios analisados, 119 alcançaram 100\% da proporção de atendimento, enquanto Mutum apresentou apenas 13,31\%, sendo este o menor valor para esta variável. Os valores de amplitude e desvio padrão demonstram que a proporção dos municípios atendidos de acordo com os não atendidos apresenta grande variação.

A Cobertura vacinal de febre amarela em menores de 1 ano apresentou média de 93,89\% em que $66,01 \%$ dos municípios estão acima da média. Obtiveram coberta máxima 127 municípios, dos 256 analisados enquanto 3 municípios apresentaram cobertura mínima (61\%).

A Proporção da população coberta por planos privados de saúde tem como média 9,19\%, sendo que $32,42 \%$ dos municípios estão acima da média. Divinópolis é o município que $55,89 \%$ da população é coberta por planos privados de saúde, enquanto em Novorizonte apenas $0,01 \%$ da população possui acesso aos planos privados. Através dos valores de amplitude e desvio padrão, nota-se que há grande discrepância quanto aos percentuais da população de cada município que são atendidos por planos de saúde privados. 
Proporção de óbito por causas mal definidas sem assistência médica tem como média $4,43 \%$, sendo que $33,2 \%$ dos municípios estão acima da média. Frei Gaspar apresentou $27,08 \%$ maior proporção de óbito por causas mal definidas, por outro lado, Governador Valadares foi o município que apresentou menor índice neste quesito $(0,12 \%)$. Com base nos valores de desvio padrão e amplitude encontrados, percebe-se uma grande discrepância entre os municípios que mais apresentam casos de óbito por causas mal definidas sem assistência médica em relação aos que apresentam menos casos.
Proporção das internações para - parto de pacientes do SUS encaminhados para outro município obteve como média $49,61 \%$ em que 46,09\% dos municípios estão acima da média. Dos 256 municípios analisados, 98 alcançaram o máximo de proporção das internações para parto de pacientes do SUS e o mínimo Unaí, sendo $0,12 \%$. São evidenciadas grandes variações com base na amplitude e desvio padrão entre os municípios, uma vez que a proporção de partos encaminhados em outros municípios oscila em detrimento de outros.

\subsection{ANÁLISE EVOLUTÓRIA DE DADOS (DEA)}

A análise envoltória dos dados foi realizada no intuito de analisar a eficiência na aplicação de recursos públicos na área de saúde. $A$ mensuração da eficiência é realizada por meio de escores que variam entre $0 \%$ e $100 \%$, sendo que os municípios eficientes receberam valor igual a 100\%. Na Tabela 3 são apresentadas as estatísticas descritivas dos escores de eficiência em comparação aos anos de 2005 e 2010 que foram obtidos com auxílio do software EMS.

Tabela 3: escores de eficiência

\begin{tabular}{llllll}
\hline Ano & Média & Máximo & Mínimo & Desvio padrão & Amplitude \\
\hline 2005 & $18,68 \%$ & $100 \%$ & $1,52 \%$ & $16,14 \%$ & $98,48 \%$ \\
\hline & & & & & \\
\hline Ano & Média & Máximo & Mínimo & Desvio padrão & Amplitude \\
\hline 2010 & $51,32 \%$ & $100 \%$ & $16,72 \%$ & $18,16 \%$ & $83,28 \%$ \\
\hline
\end{tabular}


Conforme apresentado na Tabela 3 em 2005 a média do escore de eficiência foi de $18,68 \%$ sendo que $37,11 \%$ dos municípios estão acima da média, e os municípios Campanário, Diamantina, Ibiaí e Sabará atingiram 100\% de eficiência na aplicação dos recursos. Em contraponto, Santa Vitória atingiu $1,52 \%$, o valor mínimo de eficiência. Com os valores de desvio padrão e amplitude pode-se dizer que os municípios apresentam grandes variações em relação média dos escores de eficiência.

No ano de 2010, a média de acordo com os dados coletados é de $51,32 \%$, sendo que $37,5 \%$ dos Municípios estão acima da média. Dos 256 Municípios analisados, 11 se destacam por terem atingido $100 \%$ de eficiência, enquanto Delfinópolis apresentou valor de $16,72 \%$, sendo o município com menor nível de eficiência.

Ao comparar os referidos anos, é possível afirmar que de 2005 para 2010 houve um aumento de 63,6\% na eficiência de aplicação dos recursos em saúde. Ademais, ao comparar o valor da média e desvio padrão de cada ano, conclui-se que a discrepância entre os municípios diminui consideravelmente. Destacase ainda o aumento do número de municípios com eficiência máxima os quais eram 4 em 2005, passando para 11 em 2010.

Para proporcionar melhor comparação dos níveis de eficiência, os municípios foram divididos em três grupos conforme apresentados na tabela 4:

Tabela 4: divisão dos grupos de escores de eficiência 2005

\begin{tabular}{llllc}
\hline Ano & Critério & Escores & Recursos & Grupo \\
\hline & $\begin{array}{l}\text { Inferior à Média 1 desvio } \\
\text { padrão a menos }\end{array}$ & $\mathrm{E}<34,82 \%$ & Fraco & 1 \\
\cline { 2 - 5 } 2005 & $\begin{array}{l}\text { Média mais ou menos 1 } \\
\text { desvio padrão }\end{array}$ & $34,82 \%<\mathrm{E}<50,96 \%$ & Bom & 2 \\
\cline { 2 - 5 } & $\begin{array}{l}\text { Inferior à Média 1 desvio } \\
\text { padrão a menos }\end{array}$ & E $>50,96 \%$ & Excelente & 3 \\
\hline
\end{tabular}

Com base nos valores, pode-se dizer que em $2005 \quad 91,8 \%$ dos municípios analisados apresentaram $34,82 \%$ de escore, os quais foram considerados

como fracos,

representando a má alocação dos recursos públicos na área da saúde. Dos municípios analisados 4,69\% apresentaram escores acima de 
$34,82 \%$, sendo considerados com eficiência, sendo que ainda podem ser feitas melhorias. Já os municípios que apresentam escore acima de 50,96\% representam $3,5 \%$ da amostra e foram nível

bom de considerados excelentes na alocação de recursos.

A tabela 5 apresenta a divisão dos grupos de escores de eficiência no ano de 2010.

Tabela 5: divisão dos grupos DE ESCORES de eficiência 2010

\begin{tabular}{llllc}
\hline Ano & Critério & Escores & Recursos & Grupo \\
\hline \multirow{3}{*}{2010} & $\begin{array}{l}\text { Inferior à Média 1 desvio } \\
\text { padrão a menos }\end{array}$ & $\mathrm{E}<69,48 \%$ & Fraco & 1 \\
\cline { 2 - 5 } & $\begin{array}{l}\text { Média mais ou menos 1 } \\
\text { desvio padrão }\end{array}$ & $69,48 \%<\mathrm{E}<87,64 \%$ & Bom & 2 \\
\cline { 2 - 5 } & $\begin{array}{l}\text { Inferior à Média 1 desvio } \\
\text { padrão a menos }\end{array}$ & $\mathrm{E}>87,64 \%$ & Excelente & 3 \\
\hline
\end{tabular}

Em 2010 os municípios que apresentaram escores abaixo de $69,48 \%$ foram classificados como fracos, evidenciando que os recursos públicos da saúde não estão sendo alocados eficientemente em 85,94\% dos municípios analisados. Os municípios que apresentaram escore de eficiência acima de $69,48 \%$ foram classificados com nível bom de eficiência, sendo 8,2\% dos municípios analisados, porém ainda podem ser feitas melhorias. Os que apresentaram $87,64 \%$ foram considerados excelentes na alocação de recursos perfazendo o total de $5,86 \%$ dos municípios analisados.

Ao comparar 2005 com 2010 nota-se que houve aumento dos escores, podendo então concluir que houve melhorias na alocação dos recursos públicos na área da saúde de um ano para o outro.

\subsection{TESTE DE T PARA AMOSTRAS EMPARELHADAS}

Para comprovar se houve diferença na eficiência dos municípios entre os dois períodos analisados foi realizado um teste de média para amostras emparelhadas em que foram comparados os valores dos escores de
2005 e 2010. O intervalo de confiança assumido para tomada de decisão foi de 5\%. A Tabela 6 apresenta a estatística para o teste t para amostras emparelhadas. 
Tabela 6: resultado do teste t para amostras emparelhadas

\begin{tabular}{|c|c|c|c|c|c|c|c|c|}
\hline & \multicolumn{4}{|c|}{ Diferenças emparelhadas } & \multirow[b]{3}{*}{$\mathrm{t}$} & \multirow[b]{3}{*}{ df } & \multirow{3}{*}{$\begin{array}{l}\text { Sig. (2 } \\
\text { extremidades) }\end{array}$} \\
\hline & & \multirow[b]{2}{*}{ Média } & \multirow{2}{*}{$\begin{array}{l}\text { Desvio } \\
\text { padrão }\end{array}$} & \multicolumn{2}{|c|}{$\begin{array}{l}\text { 95\% Intervalo de confiança } \\
\text { da diferença }\end{array}$} & & & \\
\hline & & & & Inferior & Superior & & & \\
\hline $\begin{array}{l}\text { Par } \\
1\end{array}$ & $\begin{array}{l}\text { Score2005 } \\
\text { Score2010 }\end{array}$ & $-32,63 \%$ & $21,37 \%$ & $-35,26 \%$ & $-30,00 \%$ & $-24,42$ & 255 & ,000, \\
\hline
\end{tabular}

Conforme pode se observar na Tabela 6 o valor da significância foi de 0,00 , e, portanto menor que o intervalo de confiança de 0,05 . Dessa forma pode-se rejeitar a hipótese nula apresentando evidencias estatísticas suficientes para afirmar que há

\section{CONCLUSÃO}

A pesquisa buscou analisar a eficiência na alocação dos recursos públicos na área da saúde nos municípios mineiros em 2005 e 2010, além de verificar o desempenho dos índices de eficiência comparando os referidos anos.

Os escores apurados através da Análise Envoltória de Dados (DEA) em 2005 demonstram situação crítica na alocação de recursos na área de saúde, pois o nível máximo de eficiência na alocação dos recursos foi alcançado em apenas 4 municípios dos 256 analisados, sendo: Campanário, Diamantina, lbiaí e diferença nos valores de eficiência para os dois períodos. Como a média aumentou de um período para o outro se pode inferir sobre a melhoria da eficiência na alocação de recursos públicos na área de saúde.

Sabará. Foram ainda apresentadas grandes variações na média dos escores de um município para outro, destacando-se Santa Vitória com 1,52\% de eficiência, sendo o município que atingiu o mínimo em eficiência na alocação dos recursos públicos na área da saúde comparado com os demais.

Em 2010, o nível de escores dos municípios aumentou em relação a 2005 63,60\%, sendo que 11 dos 256 municípios analisados atingiram 100\% de eficiência. Os resultados apontam que houve redução na variação da 
eficiência dos municípios entre os períodos analisados.

De acordo com dados obtidos da divisão dos grupos de escores, nota-se que o nível máximo de $100 \%$ de eficiência na alocação dos recursos públicos ainda é restrito a poucos municípios, o que demonstra que muito ainda deve ser feito pelos gestores mineiros.

Mesmo obtendo dados que comprovem que poucos municípios mineiros são eficientes na alocação dos recursos públicos na área da saúde, a pesquisa proposta atingiu seu objetivo que foi retratar a eficiência na alocação dos recursos públicos na área da saúde nos municípios mineiros, sendo que para mudar esses dados basta que os gestores analisem a administração dos recursos com cautela e priorizem os pontos que precisam ser melhorados, desse modo garantindo o direito a saúde a toda população que sofre constantemente com a falta de recursos públicos.

Propõem-se ainda a continuidade deste trabalho em uma abordagem qualitativa, que se baseie em analisar quais os motivos reais que levam a não eficiência e aponte melhorias que devem ser feitas para que os municípios possam ser considerados eficientes na alocação dos recursos públicos destinados a área da saúde, proporcionando assim qualidade de vida a todos os cidadãos.

\section{REFERÊNCIA}

BARROS, Fábio Batalha Monteiro. História e Legislação do SUS e Saúde da família: problematizando a realidade da saúde pública. 1 ed. Rio de Janeiro, Agbook, 2011.

BEUREN, Ilsen Maria. Como Elaborar Trabalhos Monográficos em Contabilidade. 3 ed. São Paulo: Atlas,2008.

BRASIL, Constituição Federal de 1988. Constituição da República Federativa do Brasil. Brasília, DF: Senado, 1988.

BRASIL. Lei n. 8.080, de 19 de setembro de 1990. Dispõe sobre as condições para a promoção, proteção e recuperação da Saúde, a organização e o funcionamento dos serviços correspondentes e dá outras providências. Diário Oficial da União, Brasília, DF, seção 1, p. 18055-18059, 20 set. 1990.

CABRAL, Kerla Fabiana Dias; FERREIRA, Marco Aurélio Marques. Investigação dos Níveis de Eficiência na Alocação dos Recursos Públicos Após O Pacto Pela Saúde. RAHIS, v. 11, n. 1, 2014.

KOHAMA, Hélio. Contabilidade Pública. 14 ed. São Paulo: Atlas, 2012. 
LINS, Marcos E. et al. O uso da Análise Envoltória de Dados (DEA) para avaliação de hospitais universitários brasileiros. Ciência e Saúde Coletiva, v. 12, n. 4, p. 985-98, 2007.

MARCONI, Marina de Andrade; LAKATOS, Eva Maria. Fundamentos de Metodologia Científica. 5 ed. São Paulo: Atlas, 2003.

MARINHO, Alexandre: FAÇANHA, Luis Otávio.Programas sociais: efetividade, eficiência e eficácia como dimensões operacionais da avaliação. Texto para discussão $N^{\circ}$ 805, IPEA, Distrito Federal, 2001.

MATTA, Isabela Braga. Análise do desempenho municipal da ESF à luz da eficiência relativa. Dissertação (Mestrado em Administração) - Universidade Federal de Viçosa, Viçosa 2014.

ORGANIZAÇÃO MUNDIAL DA SAÚDE. Redução das desigualdades no período de uma geração: Igualdade na saúde através da ação sobre os seus determinantes sociais. Relatório final da Comissão para os Determinantes Sociais da Saúde. Genebra: Organização Mundial da Saúde; 2010.

PESTANA, Maria Helena; GAGEIRO, João Nunes. Descobrindo a regressão: com a complementaridade do SPSS.Lisboa: Lidel, 2005.

RAMPAZZO, Lino. Metodologia Cientifica. 3. ed. São Paulo: Loyola, 2005.

RIBEIRO, Clarice de Paiva. Eficiência na Gestão dos Gastos com educação, saúde, habitação e emprego no estado de Minas Gerais. Dissertação

(Mestrado em Administração) — Universidade Federal de Viçosa, Viçosa, 2012.

SILVA, Ambrozina de Abreu Pereira.Eficiência na alocação de recursos públicos e qualidade de vida nos municípios mineiros. Dissertação (Mestrado em Administração) — Universidade Federal de Viçosa, Viçosa, 2009.

Recebido em: 25/01/2017.

Aprovado em:15/03/2017. 